\title{
Time range of logarithmic decay
}

\author{
Ivo Klik and Ching-Ray Chang \\ Department of Physics, National Taiwan University Taipei, Taiwan, 10764, Republic of China
}

(Received 23 November 1992)

\begin{abstract}
We assume a logarithmic normal distribution of activation volumes within an ensemble and study four different models of its average thermal relaxation. We extract from them a common rule for the time range on which logarithmic relaxation can be observed. We further show that to a very good approximation the coefficient of magnetic viscosity is $S_{v}=-T /(\partial\langle\mathscr{E}\rangle / \partial H)$ where $\langle\mathscr{E}\rangle$ is the average activation volume within the ensemble.
\end{abstract}

Simple theories of thermal relaxation predict an exponential-evolution law yet, as a rule, logarithmic relaxation is actually observed in measurements. Ever since the pioneering work of Street and Wolley ${ }^{1}$ and Gaunt ${ }^{2}$ it has been recognized that logarithmic relaxation arises in composite systems as an average (net) evolution law for a large number of exponentially decaying individual components. Given the great amount of both theoretical and experimental work devoted to this subject (see, e.g., a recent review of magnetic viscosity by Chantrell ${ }^{3}$ ), it is rather surprising that so far little or no attention at all has been devoted to the time range on which logarithmic decay law can be observed. We seek to ammend this situation by following the suggestion of Aharoni ${ }^{4}$ and studying, from a purely mathematical point of view, four different models of thermal relaxation and extract from them a common rule for the range of logarithmic decay. Moreover, we interpret our models as a magnetic system and derive an improved version of the relation between magnetic susceptibility $\chi_{\text {irr }}$ and magnetic viscosity $S$. In particular, we show that the coefficient of magnetic viscosity $S_{v}$ has a specific sample dependence.

Let us first consider an array of particles of activation volume $V$ which has the logarithmic normal distribution

$P(V)=(\sigma V)^{-1}(2 \pi)^{-1 / 2} \exp \left[-\left(2 \sigma^{2}\right)^{-1} \ln \left(V / V_{0}\right)\right]$

within the sample. The volume $V_{0}$ in (1) has no particular significance; the two quantities of interest are the most probable volume $V_{p}=V_{0} \xi^{-1}$ at which $P(V)$ has a maximum and the average volume $\langle V\rangle=V_{0} \xi^{1 / 2}$. The quantity $\xi=\exp \left(\sigma^{2}\right)$ was introduced here for purely typographic reasons. In the limit of $\sigma \rightarrow 0$ the distribution $P(V) \rightarrow \delta\left(V-V_{0}\right), \xi \rightarrow 1$, and $V_{p} \rightarrow\langle V\rangle$ while for large $\sigma$ the function $P(V)$ has a broad peak and $V_{p}<\langle V\rangle$. The most natural way of specifying the relaxation properties of our sample is by means of the decay rate associated with its most abundant component, i.e., with $V_{p}$, and we shall write it as $\kappa_{p}=\kappa\left(V_{p}\right)=f_{0} \exp \left(-Q_{p}\right)$. Here $f_{0}$ is a prefactor (we take it to be $e^{25} \mathrm{~Hz}$ ) and $Q_{p}=V_{p} E / T$ with $E$ being the activation energy density and $T$ the temperature $\left(k_{B}=1\right.$ throughout). For any other volume we write $\kappa(V)=f_{0} \exp \left(-Q_{p} V / V_{p}\right)$, in particular, we define $\kappa_{a}=\kappa(\langle V\rangle)$.

Let us first consider the particulate medium and as- sume that the activation volume $V$ is equal (or at least proportional) to the physical volume of the particle whose magnetic moment then becomes $M=M V$. The saturation value of the magnetization $M$ is $M_{s}$, which we assume to be temperature independent. Further let the system be prepared in the saturated state at time $t=0$ and let it be allowed to relax in zero field [nonzero field introduces, for large $Q_{p}$, only a constant factor ${ }^{2}$ into Eq. (2) below]. The magnetic moment of each particle relaxes as $M_{V}(t)=V M_{s} \exp [-\kappa(V) t]$ and the net magnetization

$$
\begin{aligned}
\langle M(t)\rangle & =\langle V\rangle^{-1}\left\langle M_{V}(t)\right\rangle \\
& =\frac{M_{s}}{\sigma(2 \pi \zeta)^{1 / 2}} \int_{0}^{\infty} d x g_{1}(x) e^{-\tau g_{2}(x)} \\
& \equiv M_{s} I_{1}^{(1)}\left(\tau \mid \sigma, Q_{p}\right) .
\end{aligned}
$$

Here we set $x=V / V_{0}$, and introduce the two functions

$$
\begin{aligned}
& g_{1}(x)=\exp \left[-\left(2 \sigma^{2}\right)^{-1} \ln ^{2} x\right], \\
& g_{2}(x)=\exp \left[-Q_{p}(x \xi-1)\right],
\end{aligned}
$$

and the dimensionless time $\tau=\kappa_{p} t$. The last equality defines the integral $I_{1}^{(1)}$. The quantities of interest are the derivatives of $\langle M\rangle$ with respect to $\ln \tau$,

$$
\begin{aligned}
\frac{\partial\langle M\rangle}{\partial \ln \tau} & =-\frac{M_{s}}{\sigma(2 \pi \zeta)^{1 / 2}} \int_{0}^{\infty} d x g_{1}(x) \tau g_{2}(x) e^{-\tau g_{2}(x)} \\
& \equiv-M_{s} I_{2}^{(1)}\left(\tau \mid \sigma, Q_{p}\right)
\end{aligned}
$$

and to the applied field $H$,

$$
\begin{aligned}
\frac{\partial\langle M\rangle}{\partial H} & =\frac{M_{s} \xi^{1 / 2}}{\sigma(2 \pi)^{1 / 2}} \frac{\partial Q_{p}}{\partial H} \int_{0}^{\infty} d x x g_{1}(x) \tau g_{2}(x) e^{-\tau g_{2}(x)} \\
& \equiv M_{s} \frac{\partial Q_{p}}{\partial H} I_{3}^{(1)}\left(\tau \mid \sigma, Q_{p}\right)
\end{aligned}
$$

The three integrals $I_{j}^{(1)}$ cannot be done analytically but are easily computed numerically. We plotted them for selected values of $\kappa_{p}$ (or equivalently of $Q_{p}$ ) and for $\sigma \in\langle 0.05,0.6\rangle$ on the interval $\tau \leq 2.5 \kappa_{p} \kappa_{a}^{-1}$, i.e., for 
times $t \leq 2.5 \kappa_{a}^{-1}$. A representative sample of these curves for $\kappa_{p}=1 \mathrm{~Hz}$ is shown in Fig. 1. In the $\tau$ parametrization their overall structure is remarkably insensitive to variations in $Q_{p}$. The function $I_{2}^{(1)}$ is almost constant for times $\tau=\rho \kappa_{p} \kappa_{a}^{-1}$, where the range of $\rho \sim 1$ increases with increasing $\sigma$. To explain this effect let us consider the extremal properties of the integrand in Eq. (5): The function $g_{1}(x)$ has a maximum at the point $x_{10}=1$ and $g_{2} e^{-\tau g_{2}}$ peaks at the point

$$
\begin{aligned}
x_{20}(\tau) & =\left(\zeta Q_{p}\right)^{-1}\left(\ln \tau+Q_{p}\right) \\
& =\zeta^{1 / 2}+\left(\zeta Q_{p}\right)^{-1} \ln \rho .
\end{aligned}
$$

Logarithmic decay is observed while the movable peak $x_{20}(\tau)$ passes through the stationary maximum of $g_{1}(x)$ at $x_{10}=1$. To see this consider first the $\sigma=0.05$ case: $g_{1}(x)$ is very sharply peaked around $x_{10}=1$ and $g_{2}$ overlaps with $g_{1}$ only on a narrow interval of $\rho$ on which $x_{10} \approx x_{20}(\tau)$. This time corresponds to the maximum of the function $I_{2}^{(1)}$ of Fig. 1. At earlier times (smaller $\rho$ ) the $g_{2}$ peak is distinct from $g_{1}$ to the left of $x_{10}$ (ascending part of $I_{2}^{(1)}$ ) and for later times it is distinct to the right (descending part of $I_{2}^{(1)}$ ). On the other hand, for large $\sigma$ the function $g_{1}$ is very broad, especially to the right of $x_{10}=1$ and the overlapping condition is easily satisfied over a longer time interval. The peak of $I_{2}^{(1)}$ then broadens accordingly but decreases its height. Equation (7) also shows that decreasing $Q_{p}$ (increasing temperature) magnifies the speed of passage of the $g_{2}$ peak through $g_{1}$ and decreases the range of logarithmic decay.

The preceeding argument constitutes the main result of this article, its corollaries are investigated below. It is interesting to note here, though, that in Gaunt's derivation ${ }^{2}$ of the formula for magnetic viscosity $S=-\partial\langle M\rangle / \partial \ln \tau$ it was assumed that the two peaks at $x_{10}$ and $x_{20}$ are far apart with $g_{1}(x)$ slowly varying and taken out of the integral as $g_{1}\left(x_{20}\right)$. This situation, however, corresponds to times far to the right of the peak of $I_{2}^{(1)}$ where this func-

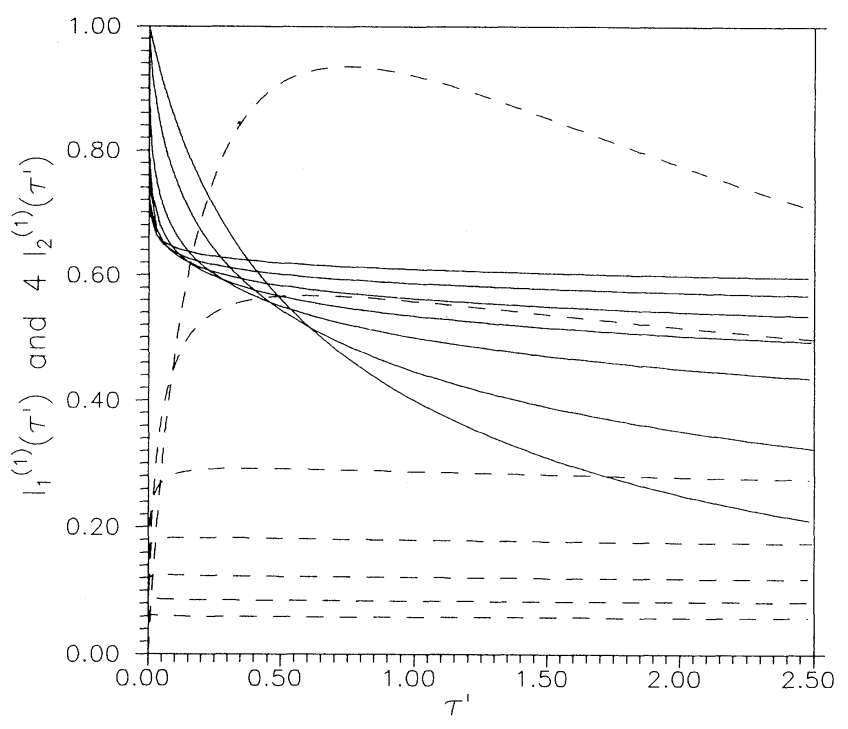

FIG. 1. The functions $I_{1}^{(1)}\left(\tau^{\prime} \mid \sigma, Q_{p}\right)$ (solid lines) and $4 I_{2}^{(1)}\left(\tau^{\prime} \mid \sigma, Q_{p}\right)$ (dashed lines) plotted vs the reduced time $\tau^{\prime}=\kappa_{p} \kappa_{a}^{-1}$. The parameter $\sigma=0.05$ and $0.1-0.6$ with increments of 0.1 . To $\sigma=0.05$ there corresponds the lowermost (fastest decaying) curve $I_{1}^{(1)}$ and the uppermost curve $I_{2}^{(1)}$ with a distinct peak. In all cases $\kappa_{p}=1 \mathrm{~Hz}$.

tion is approximately constant and almost zero. Logarothmic decay is due to the combined variation of the two functions $g_{1}$ and $g_{2}$ and is observed at the maximum of the integral $I_{2}^{(1)}$.

Having dealt with the integral (5), we shall now turn our attention to the integral (6) for $\partial\langle M\rangle / \partial H$. The two integrals have very similar structure and numerical calculations show (see Fig. 2, we were not able to demonstrate this remarkable relation analytically) that to a very good approximation $I_{3}^{(1)} \approx \xi^{3 / 2} I_{2}^{(1)}$, whence

$$
\frac{\partial\langle M\rangle}{\partial H}=\xi^{3 / 2} \frac{\partial Q_{p}}{\partial H}\left[-\frac{\partial\langle M\rangle}{\partial \ln \tau}\right] \Longrightarrow \chi_{\mathrm{irr}}=-S \frac{\langle V\rangle}{T} \frac{\partial E}{\partial H}=-S \frac{1}{T} \frac{\partial\langle\mathscr{E}\rangle}{\partial H}
$$

since $\langle V\rangle=\zeta^{3 / 2} V_{p}$. Here we associated the two derivatives of magnetization with magnetic viscosity $S$ and irreversible susceptibility $\chi_{\text {irr }}$, used our definition of the factor $Q_{p}$, and introduced the mean activation energy $\langle\mathscr{E}\rangle=E\langle V\rangle$. Equation (8) has the usual form ${ }^{1,2}$ for the relation between $\chi_{\text {irr }}$ and $S$ but we replace the customary nonstochastic expression for $\mathscr{E}$ at blocking temperature by its average value $\langle\mathscr{E}\rangle$. The coefficient of magnetic viscosity $S_{v}=S / \chi_{\text {irr }}$ is therefore sample dependent by virtue of the averaging procedure. It is satisfactory to note that Eq. (8), though based on the distribution (1), does not explicitly depend on it.

We consider next the case where the observed magnetic moment is independent of the activation volume $V$.
Such models have been used both for magnetic particles whose physical volume is unrelated to the activation volume ${ }^{5}$ and for domain-wall unpinning. ${ }^{2}$ There is then

$$
\langle M(t)\rangle=M_{s}\langle\exp [-\kappa(V) t]\rangle \equiv M_{s} I_{1}^{(2)}\left(\tau \mid \sigma, Q_{p}\right) .
$$

The modified integrals $I_{j}^{(2)}, j=1,2$, and 3, have the same structure as given in Eqs. (2), (5), and (6), but with $g_{1}(x) \rightarrow \widetilde{g}_{1}(x)=x^{-1} g_{1}(x) \xi^{1 / 2}$. Moreover, Fig. 2 shows that $I_{3}^{(2)} \approx \xi^{3 / 2} I_{2}^{(2)}$, whence Eq. (8) is immediately recovered. The modified function $\widetilde{g}_{1}(x)$ has a peak at $\widetilde{x}_{10}=\xi^{-1 / 2}$ and our plots of $I_{2}^{(2)}$ show that logarithmic decay sets in at earlier times than in the previous model. This is easily understood since $\widetilde{x}_{10}<x_{10}=1$ so that over- 


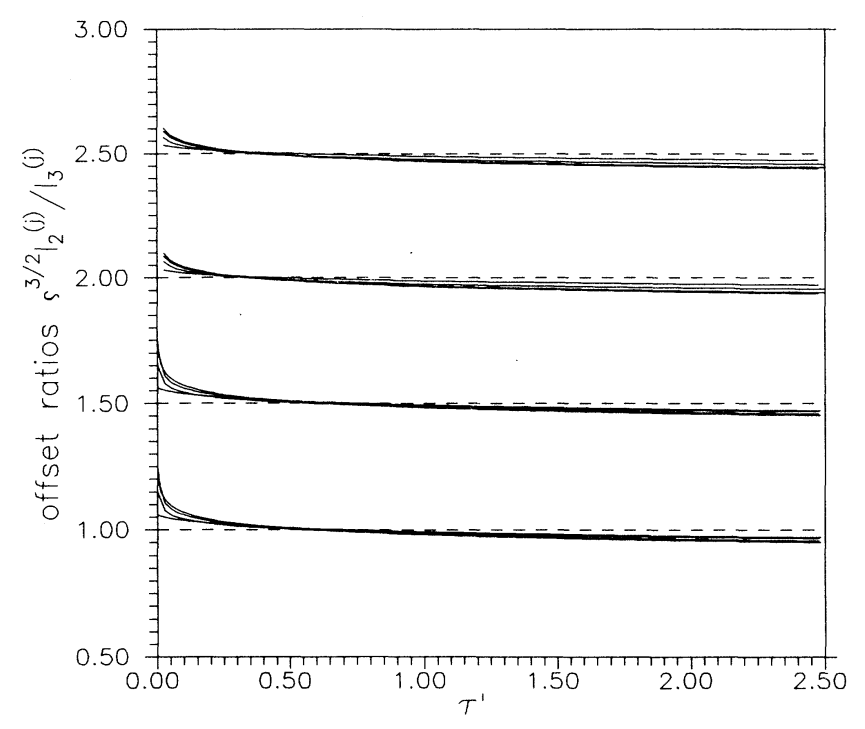

FIG. 2. The ratio $\xi^{3 / 2} I_{2}^{(j)} / I_{3}^{(j)}$ plotted vs the reduced time $\tau^{\prime}=\kappa_{p} \kappa_{a}^{-1}$. The four plots are offset along the $y$ axis by the distance $(j-1) / 2, j=1, \ldots, 4$, so that the dashed horizontal lines represent unity for each family of curves. In all cases $\kappa_{p}=1 \mathrm{~Hz}$ and selected values of $\sigma$ are in the range $\langle 0.05,0.6\rangle$. Note the excellent agreement even for $\sigma=0.05$ at which logarithmic decay has very narrow range corresponding to the peak position of $I_{2}^{(1)}$ in Fig. 1 .

lap is attained earlier [at smaller $\rho$ in Eq. (7) for the movable peak $\left.x_{20}(\tau)\right]$.

At this junction it will also be of interest to compare the time dependence of the integrals $I_{2}^{(k)}$ and $I_{3}^{(k)}$. In the integral $I_{3}^{(1)}$ the function $x g_{1}(x)$ has a maximum at $x_{30}=\xi>1$ and the movable peak of $g_{2} e^{-\tau g_{2}}$ at $x_{20}(\tau)$ overlaps with $x_{30}$ at later times than with the peak of $g_{1}$ at $x_{10}=1$ found in the integral for $I_{2}^{(1)}$. For this reason the ratio $I_{3}^{(1)} / I_{2}^{(1)}$ cannot be exactly time independent. The situation is analogous for model (9): The movable peak at $x_{20}(\tau)$ passes first through $\widetilde{x}_{10}=\xi^{-1 / 2}<1$ (in the integrand of $I_{2}^{(2)}$ ) and only later through $\widetilde{x}_{30}=1$ in the integrand of $I_{3}^{(2)}$.

In writing down Eqs. (2) and (9) we assumed that the overall evolution of an ensemble of identical relaxing components is exponential. This assumption, while being common practice, is incorrect since overbarrier relaxation is a stochastic process with mean rate $\kappa$. It is well known ${ }^{6}$ that in the asymptotic limit the first passage time $\Upsilon$ out of a metastable well has the distribution $\gamma(\Upsilon)=\langle\Upsilon\rangle \exp (-\Upsilon /\langle\Upsilon\rangle)$. For simplicity we associate ${ }^{7}$ the mean first passage time $\langle\Upsilon\rangle$ with the decay rate by setting $\kappa=\langle\Upsilon\rangle^{-1}$. The time evolution of a system of identical relaxing components is then obtained by averaging $^{7}$ the function $\exp (-t / \Upsilon)$ over the distribution $\gamma$ :

$$
\langle\exp (-t / \Upsilon)\rangle_{\gamma}=\vartheta^{1 / 2} K_{1}\left(\vartheta^{1 / 2}\right),
$$

where

$$
\vartheta=4 \kappa t
$$

and $K_{1}(z)$ is a modified (MacDonald) Bessel function. ${ }^{8}$ The function (10) has a much larger initial slope (at $\vartheta \approx 0$ ) than the simple exponent $e^{-\vartheta / 4}$ but it decays more slowly as $\vartheta \rightarrow \infty$. The integrals (2), (5), and (6) are now replaced by

$$
\begin{aligned}
\langle M(t)\rangle & =\frac{M_{s}}{\sigma(2 \pi \zeta)^{1 / 2}} \int_{0}^{\infty} d x g_{1}(x) \bar{g}_{2}(x, \tau) K_{1}\left[\bar{g}_{2}(x, \tau)\right] \\
& \equiv M_{s} I_{1}^{(3)}\left(\tau \mid \sigma, Q_{p}\right) \\
\frac{\partial\langle M\rangle}{\partial \ln \tau} & =-\frac{M_{s}}{\sigma(2 \pi \zeta)^{1 / 2}} \int_{0}^{\infty} d x_{1}(x) \bar{g}_{2}^{2}(x, \tau) K_{0}\left[\bar{g}_{2}(x, \tau)\right] \\
& \equiv-M_{s} I_{2}^{(3)}\left(\tau \mid \sigma, Q_{p}\right), \\
\frac{\partial\langle M\rangle}{\partial H} & =\frac{M_{s} \zeta^{1 / 2}}{\sigma(2 \pi)^{1 / 2}} \frac{\partial Q_{p}}{\partial H} \int_{0}^{\infty} d x x g_{1}(x) \bar{g}_{2}^{2}(x, \tau) \\
& \equiv M_{s} \frac{\partial Q_{p}}{\partial H} I_{3}^{(3)}\left(\tau \mid \sigma, Q_{p}\right)
\end{aligned}
$$

where we introduced the function $\bar{g}_{2}(x, \tau)$ $=2 \tau^{1 / 2} \exp \left[-g_{2}(x) / 2\right]$ which corresponds to $\vartheta^{1 / 2}$ of Eq. (10). Our plots show, as expected, that the initial decay is more rapid than in the preceding case and that it slows down at larger times. The extremal properties of the integrands in (11)-(13) are not easily studied analytically but within the range of logarithmic decay the peak of $\bar{g}_{2}^{2} K_{0}\left(\bar{g}^{2}\right)$ is indeed close to $x_{10}=1$. The modified integrals $I_{i}^{(4)}$ corresponding to model (9) are obtained from Eqs. (11)-(13) the same as before by the replacement $g_{1}(x) \rightarrow \widetilde{g}_{1}(x)$. Figure 2 shows that the relation $I_{2}^{(j)} \approx \zeta^{3 / 2} I_{2}^{(j)}$ is satisfied for both $j=3$ and 4 and this verifies the relation (8) also for the MacDonald evolution law (10).

In conclusion, let the temperature dependence of the integrals $I_{i}^{(j)}\left[\tau \mid \sigma, Q_{p}(T)\right]$ still be considered. We take the time $\tau=2.5 \times \kappa_{a}^{-1}$ to be representative of logarithmic decay for $\sigma \geq 0.15$ and find that on the large temperature range corresponding to $10^{-6} \mathrm{~s} \leq \kappa_{s} \leq 10^{6} \mathrm{y}$, the integrals $I_{1}^{(j)}$ decrease to a good approximation linearly with $T$ while $I_{2}^{(j)}$ (and consequently also $I_{3}^{(j)}$ ) grow as $T^{1 / 2}$. In either case the rate of change with temperature decreases with increasing $\sigma$.

One of us (I.K.) would like to thank the National Science Council of the ROC for support under Grant No. NSC-82-0208-M002-035.

(1986).

${ }^{3}$ R. W. Chantrell, J. Magn. Magn. Mater. 95, 365 (1991).

${ }^{4}$ A. Aharoni, J. Appl. Phys. 57, 4702 (1985).

${ }^{5}$ A. M. de Witte, K. O'Grady, G. N. Coverdale, and R. W.
62, 562 (1949); Proc. Phys. Soc. London, Sect. B 55, 679 (1952); 69, 1189 (1956).

2P. Gaunt, Philos. Mag. 34, 775 (1976); J. Appl. Phys. 59, 4129 
Chantrell, J. Magn. Magn. Mater. 88, 183 (1990).

${ }^{6}$ P. Talkner, Z. Phys. B 68, 201 (1987).

${ }^{7}$ I. Klik, H. L. Huang, and C. R. Chang, Proceedings of the 37th MMM Conference, Houston, Texas [J. Appl. Phys. (to be published)].

${ }^{8}$ W. Magnus, F. Oberhettinger, and R. P. Soni, Formulas and Theorems for the Special Functions of Mathematical Physics (Springer, Berlin, 1966), Sec. 3.14. 